\title{
Da avaliação à intervenção na perturbação do espetro do autismo
}

\author{
Helena Isabel da Silva Reis* \\ Ana Paula da Silva Pereira** \\ Leandro da Silva Almeida***
}

\section{Resumo}

A avaliação das perturbaçóes caracterizadas por padróes de comportamento e de desenvolvimento atípicos tal como a Perturbação do Espetro do Autismo é, no mínimo, complexa. Desde o diagnóstico à própria descrição de PEA que a contradição, incerteza e desacordo surgem entre os vários profissionais e, apesar do Diagnostic and Statistical Manual of Mental Disorders providenciar uma descrição cuidadosa do perfil sintomático da criança com PEA, a observação, a aplicação de checklists e de instrumentos standardizados, conjuntamente com a família, continua a ser crucial para o desenvolvimento de um plano individualizado de intervenção. A qualidade na elaboração dos instrumentos determina a precisão e confiança da informação obtida por estes e, quanto maior for a capacidade de informação relevante fornecida, maior a validade do instrumento e mais completa será a informação que os clínicos e pesquisadores pretendem. Apesar do aumento significativo da pesquisa sobre a PEA e dos rápidos avanços na ciência, a avaliação eficiente desta perturbação continua a ser um desafio, sobretudo, para determinar uma intervenção precoce efetiva.

Palavras-chave: Perturbação do espetro do autismo; Avaliação; Intervenção precoce.

*Professora doutora do Instituto Politécnico de Leiria, Leiria, Portugal.

** Investigadora Integrada do Centro de Investigaçăo em Educação (CIEd), Grupo de Investigaçáo Desenvolvimento Psicossocial, Aprendizagem e Necessidades Educativas Especiais, do Instituto de Educação da Universidade do Minho, Braga, Portugal.

*** Investigador Integrado do Centro de Investigação em Educação (CIEd), Grupo de Investigaçáo Desenvolvimento Psicossocial, Aprendizagem e Necessidades Educativas Especiais, do Instituto de Educação da Universidade do Minho, Braga, Portugal. 


\section{From assessment to intervention in autism spectrum disorder}

\section{Abstract}

The evaluation of disorders characterized by atypical development and behavior patterns such as the Autism Spectrum Disorder is at least complex. From diagnosis to the sole discretion of PEA that contradiction, uncertainty and disagreement arise between the various professionals and, despite the Diagnostic and Statistical Manual of Mental Disorders provide a thorough description of the child's symptomatic profile with ASD, the observation, the use of checklists and standardized tests, together with the family, remains crucial for determining an individualized plan of intervention. The design quality of instruments determines the accuracy and reliability of the information and higher capacity in relevant information provided, the greater validity of the instrument and more complete information will be obtained by researchers and clinicians as they pretend. Despite the significant increase in research on the labor force and rapid advances in science, efficient evaluation of this disorder remains a challenge, especially to determine an effective early intervention.

Keywords: Autism spectrum disorder; Assessment; Early Intervention.

Crianças com PEA são sujeitas, desde muito cedo, a avaliações sistemáticas, contudo, o processo de avaliação para este tipo de criança é tão complexo quanto desafiante, dadas as suas dificuldades sociais, de comunicação e de comportamento. A família torna-se assim um elemento chave, seja para a obtenção de informação fidedigna da criança, seja para obtenção de melhores níveis de interação entre a criança e o interlocutor (HEMMETER et al., 2001; KUHANECK; WATLING, 2010).

Neste sentido, ganha particular relevo não só a complexidade subjacente às conceçôes e práticas de avaliação em Intervenção Precoce (IP), como também, a necessidade de aprofundar formas e instrumentos de avaliação ajustados à diversidade das famílias das crianças com PEA apoiadas na IP, instrumentos esses, que deverão contemplar a participação ativa e interativa dos profissionais e das famílias de forma a tornar possível o desenvolvimento de uma visão partilhada e de uma informação holística acerca da criança (SERRANO; PEREIRA, 2011).

Avaliação, é definida como um processo para a obtenção de informação com o propósito de tomar decisões (GRISHAM-BROWN; PRETTI-FRONTCZAK, 2011). Constitui-se, muitas vezes, como o primeiro momento de contacto das famílias com a equipa de Intervenção Precoce (IP), no entanto este momento é ainda culturalmente marcado pela função técnica dos profissionais, por se considerar que a avaliação requer um domínio técnico e decisor do profissional, em detrimento da valorização do conhecimento e da participação ativa das famílias(PEREIRA; SERRANO, 2014). 
Desde os anos 80 que os profissionais e as famílias têm promovido mudanças assinaláveis na avaliação das crianças com necessidades especiais, mudanças estas que advêm das transformações vivenciadas no que são atualmente as práticas recomendadas em IP tais como abordagem centrada na família, a valorização dos contextos de vida da criança e da família e a abordagem transdisciplinar (DEC, 2014).

Segundo Division for Early Childhood (2014), a avaliação é um componente critico nos serviços que apoiam crianças com atraso ou em risco de desenvolvimento tendo como propósito o rastreio e a determinaçáo da elegibilidade dos serviços, o planeamento das intervençóes individualizadas, a monitorizaçáo do progresso da criança e os objectivos alcançados.

Algumas práticas, no processo de avaliação são recomendadas pela Division for Early Childhood (2014) sendo que a família é considerada um elemento chave em todo este processo (DEC, 2014; HEMMETER et al., 2001). Os profissionais devem trabalhar colaborativamente com a família de forma a identificar as suas preferências no processo de avaliaçáo. Ao trabalharem como equipa, os profissionais devem reunir com todos os elementos intervenientes da criança, obtendo assim o maior número de informação possível. Os materiais e estratégias de avaliação utilizados devem ser apropriados à idade e nivel desenvolvimental da criança e devem ter em atenção as carateristicas sensoriais, físicas, comunicativas, culturais, linguísticas, sociais e emocionais da criança. Os profissionais que conduzem a avaliação, devem ter a certeza que incluem todas as áreas do desenvolvimento e do comportamento de forma a identificar as forças, preferências e interesses da criança (DEC, 2014).

A avaliação deve ainda ser conduzida na língua dominante da criança, e com línguas adicionais se a criança está a aprender mais do que uma língua. Os métodos utilizados pelos profissionais devem ser variados, incluindo observaçóes e entrevistas de forma a obter informação de múltiplas formas, incluindo a família, ou outro elemento significativo na vida da criança. A informaçáo obtida deve, obrigatóriamente, ser proveniente das capacidades demonstradas pela criança nas atividades da vida diária, nas suas rotinas e ambientes naturais tais como casa, instituiçáo, centro e comunidade. Além da avaliação, os profissionais devem usar o raciocínio clinico para identificar o atual nível de funcionamento da criança e para determinar o plano de ação. A implementação da avaliação deverá ser sistemática por forma a monitorizar os progressos da criança e revisar a orientação assim que necessário. De referir, que os instrumentos de avaliaçáo utilizados devem ser suficientemente sensíveis para detetar o progresso, especialmente para as crianças com necessidades de apoio significativas. Os profissionais devem ter o cuidado de transmitir os resultados da avaliação às famílias de forma compreensiva e útil (DEC, 2014)

As práticas para a avaliação de crianças em idades precoces podem seguir a teoria desenvolvimental construtivista, que enfatiza as práticas de uma avaliação naturalista, a qual ocorre em contexto de grupo, utilizando estratégias de avaliação informais (ex.: observaçáo). Neste tipo de avaliação, os profissionais seguem os interesses da criança e obtêm a informação à medida que esta se envolve nas atividades e rotinas (ex.: os educadores em contexto de sala de aula). Estamos perante a chamada 
avaliação autêntica, isto é, o contexto onde ela ocorre, é um contexto natural para a criança e, por isso, as suas competências, conhecimento e comportamento estão elevadas ao seu mais alto nível numa exibição funcional das tarefas significativas sobre um contexto real e familiar (BAGNATO, 2008).

O que torna a avaliação autêntica, não é apenas o contexto natural em que ela decorre mas também a intervenção dos pais, educadores de infância ou outras figuras de vinculação importantes para a criança. Para Bagnato (2008), a avaliaçáo é um acontecimento essencial para as crianças e suas famílias, e os resultados deverão ser utilizados para incluir as crianças em intervençóes especializadas, que podem mudar o seu destino desenvolvimental. Dada a importância do processo de avaliação, é compreensível que os materiais e procedimentos para a avaliação da criança com PEA em IP suscitem alguns desafios porque como se sabe são crianças que têm dificuldade em generalizar as suas competências para todos os contextos.

As boas práticas da avaliação recomendadas por Bagnato (2008) alicerçam-se na abordagem centrada na família, que preconiza como princípios básicos o reconhecimento dos pontos fortes da criança e da família, a respostas às suas necessidades e prioridades e a individualizaçáo da prestaçáo de serviços e apoios, que deve ter em conta os valores, cultura e modos de vida da família. Esta abordagem considera toda a família como unidade de intervenção, reconhecendo que o bem estar de cada membro afeta os restantes e baseia-se em estratégias de identificação e resposta às necessidades da família e não apenas da criança (DUNST; TRIVETTE; HAMBY, 2007). Como cada família é una e única, com as suas próprias necessidades e prioridades, cada serviço deverá ser concebido individualmente, assegurando a melhor opção de entre as disponíveis, a efetuar pela família (MCWILLIAM, 2012).

Serrano e Pereira (2011) indicam dois critérios primordiais na abordagem centrada na família: a escolha da família e a perspetiva que parte das suas competências específicas. O primeiro critério tem como característica central o conceito de que a decisão final, relativa à criança ou à família, cabe exclusivamente a esta; quanto ao papel do profissional, deve ser de facilitador da participaçáo ativa da família na promoção das suas decisões. O segundo critério enfatiza as competências de cada família, as quais surgem das capacidades, possibilidades, valores e expectativas das mesmas; a responsabilidade do profissional é de facilitar o acesso aos recursos, através dos quais essas competências possam ser reconhecidas e utilizadas.

As boas práticas recomendadas para a qualidade da avaliação em IP cruzam-se com as linhas orientadoras que facilitam a eficiência dos programas de IP com crianças com PEA e suas famílias.

Com a avaliação da criança pretendemos obter informação válida e fidedigna capaz de fundamentar tomadas de decisão, e é em busca desta objetividade que, os testes de natureza quantitativa ou os testes referenciados a normas se apresentam particularmente relevantes. Estes testes compóem a avaliaçáo convencional e permitem tomar decisôes com uma redução dos erros de medida baseada nos resultados atingidos. É, com base nestes resultados, que os profissionais têm legitimidade para determinar o encaminhamento da criança com perturbação ou atraso desenvolvi- 
mental para serviços específicos e desenhar, em conjunto com a família, programas de intervenção individualizados (BAGNATO, 2008).

Uma das principais justificações de utilização de testes para obter o nível de desenvolvimento da criança prende-se com o seu reconhecimento como um processo concebido para aumentar o conhecimento e a compreensão das capacidades e competências da criança. Permite-nos observar possíveis alteraçóes ou variaçóes, que por si mesmas dáo origem ao aparecimento de certos padróes que, em certas idades, poderão surgir ou confirmar a presença de um diagnóstico de desenvolvimento específico (KLIN et al., 2006). Os mesmos instrumentos apresentam também algumas limitaçóes. A aparente objetividade dos scores "esconde" a subjetividade do avaliador que desempenha um determinado papel quer na administração do instrumento quer na própria cotação. Também, com o uso regular dos testes, existe uma tendência para a criança "adquirir" cotaçóes mais positivas ao longo do tempo, pois com o seu uso periódico acabam por se tornar "familiares" para a criança. Levemos ainda em consideração os contextos nos quais ocorrem as avaliaçôes das crianças. São geralmente efetuadas no território dos profissionais. Embora este contexto seja familiar e confortável para o profissional, é provável que não o seja para muitos dos pais e crianças e efetivamente, a investigação revela que o contexto no qual a avaliação ocorre também pode afetar o comportamento da criança e, desse modo, a validade dos resultados (MCWILLIAM; WINTON; CRAIS, 2003).

Reconhecendo os possíveis efeitos do contexto especialmente em crianças com PEA, o ideal seria que os programas de IP oferecessem aos pais a possibilidade de efetuarem as suas avaliaçóes em casa ou no contexto pré-escolar habitual da criança. Náo sendo possível, deve tornar-se o contexto profissional mais familiar e confortável possível para a criança e sua família. Acima de tudo, os contextos e métodos de avaliação não devem impedir o acesso das crianças aos pais ou perturbar os padróes familiares de interação entre pais e criança com PEA.

É da máxima importância que as famílias sejam membros ativos no processo de avaliação, colaborando de diversas formas em cada uma das referidas etapas; o envolvimento dos pais na avaliação de desenvolvimento dos seus filhos ajuda a desenvolver as relaçôes com os profissionais promovendo a precisão e a validade preditiva dos resultados (SERRANO; PEREIRA, 2011).

A chave para conseguir uma avaliação centrada na família é perceber as preocupaçôes, prioridades, esperanças e crenças que os pais têm acerca das suas crianças. Não há conhecimentos profissionais ou inovaçáo na metodologia avaliativa que consigam anular a necessidade desta informação (MCWILLIAM et al., 2003).

\section{Intervenção precoce na PEA: que evidências?}

O National Research Council (STANSBERRY-BRUNAHAN; COLLET-KLINGENBERG, 2010) apresenta seis recomendaçóes para o sucesso dos programas de IP em crianças com PEA: 1) A intervençáo deve começar com a maior brevidade possível a partir do momento em que se suspeita que a criança tem PEA; 2) A intervenção deve incluir o envolvimento ativo da criança com PEA em todas 
as sessões, tendo sempre presente o nível desenvolvimental e a idade da criança para que, através de atividades significativas, os profissionais consigam atingir os objetivos planeados; 3) Toda a intervenção deve estar focada nos objetivos individuais da criança com PEA, que foram delineados em conjunto com a família; 4) A intervenção deve incluir a família, incluindo o desenvolvimento das suas capacidades em lidar com a criança com PEA; 5) A intervenção deve incluir avaliações sistemáticas ao programa desenvolvido pelos profissionais e família para que o desenvolvimento da criança com PEA seja avaliado regularmente e para que o programa seja redefinido sempre que necessário; 6) As intervençôes devem incluir oportunidades inclusivas e o desenvolvimento da criança com PEA deve ser potenciado, preferencialmente nos seus contextos naturais, através das interaçóes naturais que ocorrem com as demais crianças com desenvolvimento típico (creche, jardim de infância, escola).

Qualquer programa de IP na PEA deve sustentar-se na abordagem centrada na família, partindo do princípio de que cada família tem as suas competências, que surgem das capacidades, dos talentos, das possibilidades, dos valores e das suas expectativas. Isto significa que a decisão final, no que se refere à criança ou à família, cabe exclusivamente a esta última. $\mathrm{O}$ papel do profissional no processo de decisão deverá ser o de facilitador da participação ativa da família na promoção da sua ou das suas decisôes (DUNST, 2000). Neste domínio, deve reconhecer-se que as decisóes tomadas pela família podem variar de oportunidade para oportunidade e que podem depender da perceção que a família tem não só dos seus recursos, preocupaçóes e prioridades, mas também das perceçóes daqueles que a rodeiam. Uma das responsabilidades do profissional é a de facilitar a disponibilização de meios através dos quais essas competências possam ser reconhecidas e utilizadas (DUNST; BRUDER, 2006).

Os programas de IP têm sido propostos como programas significativos para reduzir as futuras dificuldades das crianças com PEA. As linhas de orientação das melhores práticas de IP dizem que a intervenção deve começar o mais cedo possível logo que se saiba o diagnóstico (idealmente entre os 2-4 anos), utilizar avaliaçóes interdisciplinares, programas individualizados e deverão ser transversais a todos os contextos (jardim-de-infância; casa; instituição/clínica) (PAYNTER et al., 2012).

Os modelos de intervenção à PEA variam substancialmente na orientação teórica em que se sustentam, no foco de intervenção e na intensidade, no contexto em que decorrem e na evidência da eficácia dos programas elaborados. Vários autores (HEMMETER et al., 2001; MCWILLIAM et al., 2003) defendem a existência de um conjunto de práticas que devem constituir-se como "colunas dorsais" a todo o processo de apoio a crianças com PEA, nomeadamente:

- as equipas especializadas devem ser constituídas por profissionais de diferentes áreas disciplinares (educadora, psicólogo, terapeuta da fala e terapeuta ocupacional) e devem incluir os membros da família para tomar decisóes e trabalharem em conjunto;

- todos os membros da equipa (incluindo a família) devem participar ativamente na definição do Plano Individual de Intervenção Precoce (PIIP); 
- a intervenção é focada na funcionalidade, isto é, os membros da equipa devem focar-se no funcionamento da criança (ex.: envolvimento, independência, relaçôes sociais) nos seus contextos naturais;

- a equipa deverá utilizar um modelo transdisciplinar para planear e desenvolver as intervençốes;

- as prioridades da criança e da família deverão ser tomadas como objetivos a atingir pela equipa, sempre que relacionadas com a participação da criança nas AVD's;

- as rotinas da criança e da família deverão ser consideradas como oportunidades naturais de intervenção por parte da equipa;

- os profissionais deverão capacitar e corresponsabilizar as famílias para que estas apoiem o desenvolvimento dos seus filhos com a máxima competência de parentalidade, façam escolhas e tomem decisóes evitando o sentimento de "dependência" dos profissionais;

- a colaboração entre a família e profissionais deverá ser efetiva e constante para que os objetivos e os resultados pretendidos possam ser alcançados;

- as práticas de intervenção deverão ser individualizadas e sensíveis às prioridades e diversidade de cada família.

As práticas centradas na família são práticas recomendadas pela investigação com impacto em diferentes domínios da vida das famílias, nomeadamente ao nível da sua corresponsabilização, qualidade de vida e do desenvolvimento da criança (CRAIS; ROY; FREE, 2006; DUNST et al., 2007; PEREIRA, 2009; WARFIELD et al., 2004). Particularmente, no caso de crianças em idades precoces, os pais podem providenciar informação extremamente válida sobre o funcionamento do seu filho com PEA no contexto de casa e da comunidade, que seria extremamente difícil, se náo impossível de obter por um outro elemento da equipa.

Uma das grandes dificuldades das crianças com PEA, é a generalização das capacidades apreendidas e, por isso, competências demonstradas em determinados contextos náo são, a maioria das vezes, observados noutros, pelo que as famílias se tornam os "membros da equipa" que melhor podem descrever as capacidades, desafios e história desenvolvimental da sua criança (BOYD et al., 2010). A família, ao partilhar a clara compreensão, os interesses e capacidades da sua criança com PEA, pode ajudar os profissionais na tomada de decisão sobre o tipo de avaliação mais apropriada e sobre o plano de intervençáo a desenvolver, apoiando e colaborando em todo o processo de avaliação - intervenção da criança.

A família, ao ser o elemento mais próximo da criança por excelência, é também o elemento mais capaz de obter melhores níveis de interação com a criança com PEA e alcançar o seu melhor nível de funcionamento (BAGNATO, 2008). 


\section{Abordagens utilizadas na intervenção às PEA}

O impacto de vários programas de intervenção junto de crianças com PEA em idades muito precoces tem sido foco de intensa investigação nos últimos anos. (ROGERS; VISMARA, 2008).

A importância de clarificar as diferentes abordagens de intervençáo específicas para as PEA tem sido estudada no sentido de permitir uma opção mais fundamentada, por parte das famílias e profissionais, na escolha de um programa de intervenção (CENTER, 2011; HOWLIN; MAGIATI; CHARMAN, 2009; HUME et al., 2011; WARREN et al., 2011).

Magyar (2011) sugere que a organização das diferentes abordagens de intervençáo se pode organizar da seguinte forma: abordagens desenvolvimentais; abordagens comportamentais; abordagens com base em terapias, entre outras.

\section{Abordagens desenvolvimentais}

Neste tipo de abordagens o foco é a construção, por parte da criança, de relaçôes positivas e significativas com os outros e o desenvolvimento de competências sociais. São abordagens estruturadas tendo como modelo o desenvolvimento típico da criança. A criança é percecionada a partir das competências que emergem ao longo do tempo e são anotados os desvios ao desenvolvimento considerado típico. A intervenção, nestes modelos, tem em consideração a interação entre as competências da criança e os ambientes que a envolvem. Muitas destas abordagens têm como objetivos promover a atenção, relacionando com a interaçáo com os outros e contribuir para a organização do pensamento lógico (ROBERTS; PRIOR, 2006).

Para Magyar (2011), as abordagens desenvolvimentais apresentam as seguintes características:

- Têm em consideração todas as áreas do desenvolvimento;

- O planeamento da intervenção integra a informaçáo sobre a criança do ponto de vista biológico, cognitivo, emocional e social;

- A criança é considerada como um ser único e com um perfil idiossincrático;

- O alvo são os aspetos negativos da perturbação que afetam o desenvolvimento;

- A criança tem um papel importante no seu próprio desenvolvimento;

- A intervenção segue o perfil de desenvolvimento considerado típico e por isso adequa-se à maioria dos currículos educativos;

- A intervenção foca-se no "ensinar" a criança a auto regular-se.

São exemplo das abordagens desenvolvimentais o programa Developmental Social Pragmatic Model (DSP); o Programa DIR/Floortime e o Programa Relationship Developmental Intervention (RDI). 


\section{Abordagens comportamentais}

Nestas abordagens, as PEA são percebidas em termos do comportamento ou áreas de competência e é feita a análise do comportamento excessivo, inapropriado ou alterado. Estas abordagens estão relacionadas com métodos que interligam contingências ambientais e comportamento. $\mathrm{O}$ comportamento alvo é identificado, a intervenção é aplicada e avaliada para verificar a adequação da intervenção.

Segundo Magyar (2011) as abordagens comportamentalistas apresentam as seguintes características:

- aplicam-se a diferentes áreas com o objetivo de desenvolver repertórios de competências;

- há uma sequência sistemática na intervenção;

- o alvo da intervenção é definido sequencialmente;

- a criança tem um currículo individualizado;

- é feita avaliaçáo regular para adequar ou modificar a intervenção;

- as atividades são estabelecidas em alternância tendo em conta propostas do adulto ou seguindo os interesses da criança;

- a intervenção tem como objetivo a generalização das competências em diferentes lugares e com diferentes pessoas.

São exemplo desta abordagem os programas Applied Behaviour Analysis (ABA); Early Intensive Behavioural Interventions (EIBI/ IBI) e o Contemporary ABA. O National Professional Development Center on Autism Spectrum Disorders aponta que é possível aumentar os comportamentos apropriados em crianças com PEA em idades muito precoces, através da Intervenção Comportamental, adotando uma orientação teórica baseada na análise do comportamento (Applied Behavior Analisys) e induzindo o comportamento esperado através do reforço e análise de tarefas (BOYD et al., 2010). No entanto, ainda não existem estudos publicados que certifiquem a eficácias das intervençóes comportamentais intensivas precoces contudo, acredita-se que algumas crianças em idades mais avançadas beneficiam de uma intervenção baseada nestas práticas comportamentais (BOYD et al., 2010; BUTTER; WYNN; MULICK, 2003).

\section{Abordagens com base nas terapias}

Neste tipo de intervenção o foco da aplicação é o desenvolvimento da comunicação social ou sensorial. São normalmente utilizadas como complemento de outras intervençôes.

Podem ter uma vertente direcionada para a comunicação e podemos referir os Programas Visual Supports/ Alternative and Augmentative Communication (AAC), o Programa Picture Exchange Communication System (PECS), o Programa Facilitated Communication (FC) e o Functional Communication Training (FCT); ou uma verten- 
te mais direcionada para os aspectos sensoriomotores e nestes podemos enquadrar o Programa Auditory Integration Training (AIT), o Programa Sensory Integration ou o método Doman-Delecato (Salvadó-Salvadó, et al., 2012).

Uma das abordagens neste tipo de terapias são os Suportes Visuais, os quais têm sido considerados "pistas" fundamentais com o propósito de ajudar as crianças com PEA a um maior envolvimento no ambiente e uma forma destas crianças apresentarem comportamentos mais adaptativos perante as rotinas diárias. (BOYD et al., 2010; WETHERBY; PRIZANT; SCHULER, 2000).

De igual modo, o Sistema de Comunicação Aumentativa e Alternativa (SAAC), é um sistema utilizado nestas terapias para definir outras formas de comunicação quando existe comprometimento da comunicação oral. Neste sistema, estão assim incluídas formas de comunicação como o uso de gestos, sinais, expressões faciais, o uso de pranchas de alfabeto ou símbolos pictográficos, ou mesmo o uso de sistemas sofisticados de output com voz sintetizada (GLENNEN; DECOSTE, 1997). Este sistema permite que as crianças com dificuldades na comunicação, como as crianças com PEA, encontrem uma forma alternativa e aumentativa de comunicar, permitindo assim a transmissão de uma mensagem ao interlocutor (WERTSCH; DEL RÍO; ALVAREZ, 1995).

Os sistemas de símbolos gráficos são os mais utilizados junto de crianças com PEA e compreendem desde as tabelas simples de apontar até aos equipamentos baseados em suportes informáticos que armazenam os símbolos e ajudam à sua transmissão. A técnica de utilizaçáo do sistema de símbolos é variável consoante as necessidades da criança e, dentro do quadro das PEA, existem essencialmente duas abordagens mais utilizadas: Picture Exchange Communication System (PECS) e os Simbolos Pictográficos para a Comunicaçáo (SPC). A diferença dos dois sistemas reside na forma de implementação de cada um por parte dos profissionais.

Face à exiguidade de instrumentos que avaliem e monitorizem os programas de intervenção na área das PEA, torna-se útil e oportuno a construção e validação de instrumentos orientados para este objetivo, para que as equipas possam comparar o "antes e o depois" relativamente às competências adquiridas pela criança nas áreas de desenvolvimento comprometidas e as evoluçôes sentidas após a intervenção de equipas especializadas. Não menos importante, será a participação da família no processo da avaliação que neste artigo contemplamos, como elementos mais próximos e conhecedores da criança, por excelência.

\section{Referências}

BAGNATO, S. Authentic assessment for early childhood intervention: Best practices. New York: Guilford Press, 2008. ISBN 1606232509.

BOYD, B. et al. Infants and toddlers with autism spectrum disorder: Early identification and early intervention. Journal of Early Intervention, v. 32, n. 2, p. 75, 2010.

BUTTER, E.; WYNN, J.; MULICK, J. Early intervention critical to autism treatment. Pediatric annals, v. 32, n. 10, p. 677-684, 2003.

CENTER, N. A. Evidence-based pratice and autism in the schools: a guide to providing appropriate interventions to studentes with autism spectrum disorders. Massachusetts: NAC, 2011. 
CRAIS, E. R.; ROY, V. P.; FREE, K. Parent's and professional's perceptions of the implementation of familycentered practices in child assessments. American Journal of Speech-Language Pathology, v. 15, n. 4, p. 365-374, 2006.

DEC. Division for Early Childhood recommended practices in early intervention/early childhood special education. v. 2014, 2014.

DUNST, C. Revisiting "Rethinking Early Intervention”. Topics in Early Childhood Special Education, v. 20, n. 2, p. 95-104, 2000.

DUNST, C.; BRUDER, M. Advancing the agenda of service coordination. Journal of Early Intervention, v. 28 , n. 3, p. 175-177, 2006.

DUNST, C.; TRIVETTE, C.; HAMBY, D. Meta-analysis of family-centred helpgiving practices research. Mental Retardation and Developmental Disabilities Research Reviews, v. 13, n. 14, p. 370-378, 2007.

GLENNEN, S.; DECOSTE, D. The handbook of augmentative and alternative communication. San Diego: Singular Publishing Group, 1997. ISBN 1565936841.

GRISHAM-BROWN, J.; PRETTI-FRONTCZAK, K. Assessing Young Children in Inclusive Settings: The Blended Practices Approach. ERIC, 2011.

HEMmeTER, M. et al. DEC Recommended Practices Program Assessment: Improving Practices for Young Children with Special Needs and Their Families. Missoula: Division for Early Childhood, 2001. ISBN 1570354855.

HOWLIN, P.; MAGIATI, I.; CHARMAN, T. Systematic review of early intensive behavioral interventions for children with autism. American Journal on Intellectual and Developmental Disabilities, v. 114, n. 1, p. 23-41, 2009.

HUME, K. et al. Assessing implementation of comprehensive treatment models for young children with ASD: Reliability and validation of two measures. Research in Autism Spectrum Disorders, v. 2, p. 1-11, 2011.

KLIN, A. et al. Avaliação clínica de crianças com risco de autismo. Educaçáo, v. 29, n. 1, p. 255-297, 2006.

KUHANECK, H. M.; WATLING, R. Autism: A comprehensive occupational therapy approach. Bethesda: AOTA PRESS, 2010.

MAGYAR, C. I. Developing and Evaluating Educational Programs for students with autism. Rochester: Springer, 2011.

MCWILLIAM, P. J.; WINTON, P. J.; CRAIS, E. R. Estratégias práticas para a intervençáo centrada na família. Porto: Porto Editora, 2003.

MCWILLIAM, R. Trabalhar com as famílias de crianças com necessidades educativas especiais. Porto: Porto Editora, 2012.

PAYNTER, J. et al. A Pilot Study of the Effects of an Australian Centre-Based Early Intervention Program for Children with Autism. Open Pediatric Medicine Journal, v. 6, p. 7-14, 2012.

PEREIRA, A. P. Práticas centradas na família em intervençáo precoce: Um estudo nacional sobre práticas profissionais. 2009. Tese de Doutoramento Instituto de Educação, Universidade do Minho

PEREIRA, A. P.; SERRANO, A. M. Early Intervention in Portugal: Study of professionals perceptions. Journal of Family Social Work, v. 17, n. 3, p. 263-282, 2014.

ROBERTS, J. M.; PRIOR, M. A review of the research to identify the most effective models of practice in early intervention of children with autism spectrum disorders. Australia, 2006.

ROGERS, S.; VISMARA, L. Evidence-based comprehensive treatments for early autism. Journal of Clinical Child \& Adolescent Psychology, v. 37, n. 1, p. 8-38, 2008.

SALVADÓ-SALVADÓ, B.; et al. Modelos de intervención global en personas con trastorno del espectro autista. Revista de Neurologia, v. 54, (Supl 1), 2012, pp 63-71.

SERRANO, A. M.; PEREIRA, A. P. Parâmetros recomendados para a qualidade da avaliação em intervenção precoce. Revista Educaçáo Especial, v. 24, n. 40, p. 163-180, 2011.

STANSBERRY-BRUNAHAN, L. L.; COLLET-KLINGENBERG, L. L. Evidence-based practices for young children with autism spectrum disorders: guidelines and recommendations from the national resource council and national professional development center on autism spectrum disorders. International Journal of Early Childhood Special Education, v. 2, p. 45-56, 2010. 
WARFIELD, M. E. et al. The effect of early intervention services on maternal well-being. In: FELDMAN, M. A. (Ed.). Early intervention: The essencial readings. Malden: Blackwell Publishing, 2004. p. 285-308.

WARREN, Z. et al. Therapies for children with autism spectrum disorders. Rockville: Agency for Healthcare Research and Quality, 2011.

WERTSCH, J.; DEL RÍO, P.; ALVAREZ, A. Sociocultural studies of mind. Cambridge: Cambridge University Press, 1995. ISBN 0521476437.

WETHERBY, A.; PRIZANT, B.; SCHULER, A. Understanding the nature of communication and language impairments. Autism Spectrum Disorders: A transactional Developmental Perspective, v. 9, p. 109-141, 2000.

\section{Correspondência}

Helena Isabel da Silva Reis - Rua do Tratado de Tordesilhas no 252, 4425-697, Pedrouços-Maia - Portugal.

E-mail: helena.s.reis@ipleiria.pt - appereira@ie.uminho.pt - leandro@ie.uminho.pt

Recebido em 08 de setembro de 2015

Aprovado em 13 de novembro de 2015 